ALVARADO, Agustina, "Recensión: GATTA, Gian Luigi, La minaccia. Contributo

allo studio delle modalità della condotta penalmente rilevante,

Roma: Aracne, 2013, 313 páginas."

Polít. crim. Vol. 9, No 18 (Diciembre 2014), Rec. 2, pp. 820 - 824.

[http://www.politicacriminal.cl/Vol_09/n_18/Vol9N18R2.pdf]

\title{
Recensión: GATTA, Gian Luigi, La minaccia. Contributo allo studio delle modalità della condotta penalmente rilevante. «I libri» di Archivio penale 20, Roma, Aracne, 2013, 313 páginas.
}

Agustina Alvarado Urízar

Abogada Universidad Católica de Valparaíso

(c) Doctor en Derecho Penal y Procesal Penal

Università degli Studi di Milano

1. Previo a efectuar una descripción general de la obra con indicación de sus aspectos relevantes para la discusión jurídica nacional, es oportuno situar el actual estado de la incidencia práctica en que se encuentra el delito de amenazas en Chile. En efecto, las estadísticas en materia de criminalidad por categoría de delito bajo el título de "delitos contra la libertad e intimidad de las personas", si bien no se arroja una cifra concreta atinente al delito de amenazas, permiten entrever un resultado no despreciable de judicialización, cercano a poco más del diez por ciento de causas ingresadas al sistema. Esta observación debe conjugarse con la repercusión que este tipo penal despliega bajo la vigencia de la Ley $\mathrm{N}^{\circ} 20.066$ de "violencia intrafamiliar", donde - según información estadística del Ministerio Público - el delito de amenazas representa el 39,10\% del total de causas a nivel nacional en esta materia. Por ende, los aportes que contribuyan a dilucidar la correcta aplicación del tipo de amenazas constituyen un tópico no sólo bienvenido sino además necesario en atención a su cuantiosa aplicación. Así, no obstante la diversa estructuración típica que ambos ordenamientos, italiano y chileno, prevén para este título de castigo, el trabajo en análisis reviste interés por traer a colación similares conclusiones a las que ya la doctrina y jurisprudencia de nuestros tribunales han esbozado en torno a la distinción entre amenaza e intimidación, pero sobre todo, por desarrollar criterios interpretativos restrictivos de aquella amenaza de un mal no constitutivo de delito, respecto de la cual nuestro medio no ha detenido mayormente la mirada. La investigación dirigida por el autor busca directrices contenedoras del tipo de amenazas desde una perspectiva de conjunto, con particular análisis de la regulación civil a partir de lo dispuesto en los arts. 1434 y siguientes del código civil italiano, que en lo pertinente, se asimilan al contenido del art. 1456 del código civil chileno, de modo que las condiciones materiales otorgan un escenario común sea para replantear una extrapolación de tales conclusiones, sea para replicar el modo de análisis, según la propia convicción.

2. Tal como el autor consigna en la primera línea de su premisa, el título de la obra indica inmediatamente su objeto. El trabajo investigativo aborda dos aspectos que materializan un fenómeno que ciertamente escapa al delito de amenazas y que va más allá de la tradición jurídico penal italiana. En efecto, en base a la redacción del tipo base de amenaza del art. 612 y 610 codice penale italiano y a la aplicación jurisprudencial de la figura, el autor advierte una huida a interpretaciones fuertemente extensivas del concepto de amenaza que no sólo lo espiritualiza, sino que además termina por desnaturalizar la función selectiva del tipo respectivo. De la importancia sustancial de esta tendencia, y 
ALVARADO, Agustina, "Recensión: GATTA, Gian Luigi, La minaccia. Contributo

allo studio delle modalità della condotta penalmente rilevante, Roma: Aracne, 2013, 313 páginas."

teniendo presente su cuantitativa repercusión desde el punto de vista de los numerosos tipos delictivos de la parte especial que la contienen, el autor se plantea la exigencia de construir los lineamientos necesarios para una interpretación restrictiva del término "amenaza", respetuosa del principio de ofensividad de bienes jurídicos (que como se verá identifica en la integridad psíquica y en la libertad de autodeterminación del sujeto) y del principio de fragmentariedad en cuanto manifestación de la opción socio-política y legislativa de su consagración. He aquí donde se advierte la particularidad del trabajo constructivo, y que lo dota de una dimensión interesante a partir de un análisis del todo innovador: la búsqueda de una interpretación restrictiva del tipo, o más precisamente, de la modalidad de ejecución de la conducta, apuntando a la alineación de un concepto unitario de amenaza, transversal a toda la parte especial y tendencialmente válido para el entero ordenamiento jurídico en general, con particular sintonía con el origen y regulación civil de la misma.

3. La obra se estructura en dos partes. La primera parte titulada "La minaccia nell'ordinamento giuridico: tra tutela dell'integrità psichica e della libertà di autodeterminazione", que consta, a su vez, de tres capítulos que en términos generales están destinados a clasificar los tipos de amenaza según su contenido (amenaza-fin o pura y simple [art. 612 c.p.i] y la amenaza-medio [art. 610 c.p.i]), en relación al bien jurídico protegido por cada una de ellas (integridad psíquica como sostén base de toda amenaza con la consecuente precisión de la libertad de autodeterminación en el caso de la amenaza condicional), todo en un interesante confronto entre la regulación civil y penal de tales manifestaciones. Ya del primer capítulo "Inquadramento preliminare e tipologie fondamentali: minaccia-fine e minaccia-mezzo" es posible extraer ideas claves para comprender la dirección en que se encamina el autor, quien enuncia una premisa fundamental, que tal vez por su obviedad se desatiende en la argumentación aplicativa de la figura, y que consiste en la equiparación de la amenaza en cuanto vis animo illata, con la violencia como vis corpori, como modalidades de ofensa típica de agresión en contra de un sujeto. En este sentido, el autor repara en la capacidad de intimidación de la conducta en cuanto idónea para producir temor, como primera aproximación para la selección de un comportamiento penalmente relevante, común a toda amenaza. Para explicar el grado de intensidad al que se apunta, el autor distingue la ofensa a la integridad psíquica de la amenaza simple materializada en el temor que suscita en el sujeto pasivo, del efecto de coacción propio de la amenaza medio. En efecto, es en relación con este último extremo donde se inicia una concatenación argumentativa global que enlaza la regulación civil del fenómeno: la amenaza condicionada dirigida a coartar la voluntad de la víctima para obtener un cierto resultado es la que encuentra consagración en el ordenamiento civil como amenaza relevante constitutiva de vicio del consentimiento y causa de anulabilidad de los contratos (art. 1434 y siguientes c.c.i.), y es la modalidad de conducta que integra los numerosos tipos pluriofensivos de la parte especial. Bajo este hilo conductor, el Capítulo segundo referido a "La minaccia nel diritto civile" contribuye a enarbolar la libertad de autodeterminación como bien jurídico protegido por la amenaza condicional, la que en concepto del autor se deduce de la identificación de subespecies de la misma extensamente tratadas en sede civil, a saber: la tutela de la libertad contractual, la tutela del poseedor y la regulación de la amenaza como ilícito civil susceptible de engendrar responsabilidad extracontractual (art. 2043 c.c.). En cada una de ellas se exponen sugestivas observaciones 
que el autor estima relevantes desde la perspectiva penal de la cuestión, de las que cabe destacar, por una parte, el diverso tratamiento que la tutela de la libertad de contratación contempla en términos de la nulidad/inexistencia y anulabilidad de los actos jurídicos en atención a la magnitud que revista la amenaza-vicio que los afecte, según se dirija a aniquilar (manifestación de vis absoluta) o sólo mermar la capacidad de autodeterminación del sujeto, lo que a su parecer, permite entrever una pretensión intrasistémica de concebir sanciones de diversa gravedad en correlación a la entidad propia de la ofensa; y por otra, en lo que respecta a la tutela de la determinación del poseedor a partir del despojo violento (físico o moral) de la propiedad (art. 1163 c.c.), la notable - aunque breve - referencia que el autor efectúa a la figura típica de la rapina mediante amenaza, enfatizando la particular intensidad que debe asumir el efecto coactivo en el terreno penalístico. El tercer y último Capítulo de este apartado trata derechamente "La minaccia nel diritto penale", donde el autor, sin pretender efectuar una enumeración completiva, efectúa una clasificación de las funciones que pueden asumir las figuras bases de amenaza (simple [art. 612 c.p.] y condicional [art. 610 c.p.]), esto es en cuanto elementos constitutivos de otros ilícitos que dan lugar a estructuras complejas, o bien como circunstancias agravantes, desempeñando el mismo rol atribuido en ocasiones a la violencia, todo con el objeto de iluminar la aptitud y la dirección ofensiva de la conducta en virtud del común denominador representado por la ofensa a la integridad psíquica y la libertad de autodeterminación. De este modo, el autor ofrece un recorrido por diversas figuras delictivas en relación de especialidad con las figuras bases ya anotadas, y se detiene a considerar los diversos intereses involucrados que explican las diferencias penológicas de las respectivas conminaciones.

4. La segunda parte de la obra rubricada "Requisiti di rilevanza giuridica della minaccia: diritto penale e civile a confronto", se embulle totalmente en el objetivo de la investigación sobre la base de las conclusiones expresadas en la primera (p. 90 y ss.). El esquema adoptado por el autor para abordar con éxito el punto puede sintéticamente describirse, bajo el prisma de la tipicidad de la conducta, en el análisis por capítulos destinados: a la identificación de los sujetos que pueden asumir el rol activo y pasivo de la figura (Capítulo IV); y a la determinación del objeto de la conducta, estos es, los presupuestos de existencia del "mal o daño injusto" (Capítulo V), y las características que debe reunir este último para ser jurídicamente relevante (Capítulo VI). Así, en el primer apartado mencionado, bajo el título "La minaccia come fatto umano che ingenera metus ab extrínseco", el autor fija como punto de partida la relación causa/efecto con que necesariamente debe concebirse el binomio amenaza/temor, que en caso alguno pueden confundirse ni fundirse en modo de identificar una amenaza en cada caso que se constate la existencia de temor. Con ello esboza el parámetro general dentro del cual debe desarrollarse el contenido de los requisitos de una amenaza penalmente relevante - desde una perspectiva restrictiva -, el cual consiste en la diferenciación del metus ab intrinseco del metus ab extrinseco, limitando sólo a estos últimos el ámbito operativo de la figura típica, y siempre que la misma provenga de la conducta humana. Se trata de cuestiones que, prima facie parecen revestidas de singular simplicidad, pero que en el trabajo sucesivo juegan un importante canal de dirección en la labor del intérprete, y que despliegan sus consecuencias en los restantes apartados. Especial relevancia revisten sus observaciones en materia de entes y personas jurídicas, así como la problemática de amenazas relativas al suicidio y otros actos autolesivos, donde el rol de agente y de bersaglio recaen en el mismo sujeto. El 


\section{ALVARADO, Agustina, "Recensión: GATTA, Gian Luigi, La minaccia. Contributo allo studio delle modalità della condotta penalmente rilevante, Roma: Aracne, 2013, 313 páginas."}

Capítulo V "La minaccia e il suo oggetto: il male o danno ingiusto" exhibe casos jurisprudenciales sobre la determinación de un supuesto mal injusto, que pueden resultar exorbitantes en nuestra óptica nacional, pero que son meritorios de análisis desde una perspectiva preventiva de aproximación al tema. En efecto, se trata por ejemplo la amenaza de terminar una relación sentimental o de amistad, o de continuidad en la pertenencia a un grupo, y se incorpora un interesante debate en torno al estudio de la amenaza por omisión y de la amenaza a través de una omisión. Por su parte, a propósito de la injusticia del mal amenazado se comprende la alusión a la relación entre el medio y el objetivo de la conducta desde la perspectiva de la justicia e injusticia de cada uno de ellos. Se trata en definitiva, por una parte, de determinar el confín entre la amenaza penalmente relevante de otras molestias meritorias de tipificar bajo un título de castigo diverso, de menor entidad; y, por otra parte, de diferenciarla de conductas abusivas o de aprovechamiento, independientemente de que éstas últimas, a su vez, puedan integrar nuevas figuras. Por último, en el Capítulo VI "Forma e attitudine ofensiva della minaccia", con ocasión de la "forma" se aborda el tema de la amenaza implícita en relación con el requisito base de exteriorización de la conducta (metus ab extrinseco como mínimo comportamiento conminatorio), en conjugación con las exigencias de prueba procesal correspondiente y con la coyuntural presencia de la criminalidad organizada, como fenómeno de corte mafioso latente. En este orden de ideas se analizan las hipótesis de temor interno o auto-procurado, la amenaza putativa, el temor reverencial y la llamada amenaza ambiental. Luego, en cuanto a la "aptitud ofensiva", el autor se detiene en la construcción de los requisitos de idoneidad constituidos por la credibilidad/seriedad de la amenaza y su gravedad, los cuales, a su parecer, debiesen predicarse simultáneamente respecto del mal o daño injusto para arribar finalmente al verdadero sustrato de la amenaza penalmente relevante. Si bien estos últimos aspectos coinciden con el panorama vigente entre nosotros, contribuyen a enriquecer el bagaje argumentativo, a extenderlo a las amenazas de un mal no constitutivo de delito y a fijar en la retina interpretativa aspectos que en ocasiones, aunque sabidos, se soslayan en las fundamentaciones jurisprudenciales.

5. De este modo, como ya he advertido, la obra comentada representa un trabajo de sumo interés en el quehacer interpretativo de las amenazas propiamente tales, y expone un amplio panorama de las repercusiones que la discusión suscita en la parte especial del derecho penal italiano. En este sentido, el trabajo analizado representa una contribución adicional al debate nacional en cuanto incentiva, a mi parecer, por una parte, la revisión de una serie conceptos tales como la determinación de la concurrencia o ausencia del consentimiento de la mujer en el tipo de aborto, donde precisamente se ha discutido que la norma incriminadora se refiera a la regulación civil del mismo; la definición de una línea demarcatoria entre intimidación y amenazas con las respectivas consecuencias en la extensión de los tipos de extorsión, robo y en delitos sexuales, y en la elaboración del contenido de la ocupación o usurpación "no violenta"; y por otra, una racional mirada hacia la tipificación de nuevas figuras en materia de corrupción en los términos en que la misma es concebida en nuestro país, y el desvanecimiento de la pretensión de penalizar otras. De ahí la expresa recomendación de estudio de la presente investigación, que a partir de un notable trabajo de análisis de jurisprudencia, pertinentes referencias procesales, y metódica 
Polít. crim. Vol. 9, No 18 (Diciembre 2014), Rec. 2, pp. 820 - 824.

[http://www.politicacriminal.cl/Vol_09/n_18/Vol9N18R2.pdf]

utilización de abundante material bibliográfico alemán e italiano, sin duda contribuirá a la construcción de nuevas perspectivas tanto de forma como de fondo. 